\title{
Bringing up to date the French database of nuclear workers contaminated with plutonium and/or americium and treated with Ca-DTPA
}

\author{
Stephanie Lamart ${ }^{1, *}$, Lise Carbone $^{2}$, Laurence Jossoud ${ }^{3}$, Philippe Bérard ${ }^{3}$, Xavier \\ Castagnet $^{4}$, Jean-Vincent Dauteuil ${ }^{3}$, Gérald Gagna ${ }^{5}$, Valérie Juve ${ }^{6}$, Josseline Lecoix ${ }^{7}$, \\ Yannick Lecompte ${ }^{5}$, Dominique Leray ${ }^{8}$, Xavier Millot ${ }^{9}$, Isabelle Puech ${ }^{10}$, Mariette Ruffin ${ }^{11}$, \\ Michel Tricotti ${ }^{12}$, and François Pic $^{3}$ \\ ${ }^{1}$ Laboratoire de Radio Toxicologie, CEA, Université Paris-Saclay, 91297 Arpajon, France \\ ${ }^{2}$ Service de Santé au Travail, CEA, 30207 Bagnols-sur-Cèze, France \\ ${ }^{3}$ Cellule Conseiller Médical, CEA, 92265 Fontenay-aux-Roses, France \\ ${ }^{4}$ Service de Santé au Travail, CEA, 33116 Le Barp, France \\ ${ }^{5}$ Service de Protection Radiologique des Armées, 92190 Clamart, France \\ ${ }^{6}$ Service de Santé au Travail, CEA, 13108 Saint-Paul-les-Durance, France \\ ${ }^{7}$ Laboratoire de biologie médicale, CEA, 30207 Bagnols-sur-Cèze, France \\ ${ }^{8}$ Service de Santé au Travail, ORANO, 50440 Beaumont Hague, France \\ ${ }^{9}$ Laboratoire de biologie médicale, CEA, 91191 Gif-sur-Yvette, France \\ ${ }^{10}$ Service de Santé au Travail, CEA, 21120 Is-sur-Tille, France \\ ${ }^{11}$ Laboratoire de biologie médicale, CEA, 13108 Saint-Paul-les-Durance, France \\ ${ }^{12}$ Laboratoire de biologie médicale, CEA, 21120 Is-sur-Tille, France
}

The diethylenetriaminepentaacetic acid (DTPA) is a chelating agent used to decorporate plutonium $(\mathrm{Pu})$, americium $(\mathrm{Am})$ and curium $(\mathrm{Cm})$ after internal contamination. The DTPA-actinide chelate is mainly excreted in urines. Hence, DTPA decorporation therapy increases and accelerates actinide excretion, which results in a decrease in the actinide systemic retention. In France, Ca-DTPA has been used since 1962 and authorized by the French National Agency for Medicines and Health Products Safety (ANSM) since 2008 [1]. This authorization has been given upon the completion of a first study of French nuclear workers contaminated with $\mathrm{Pu}$ and/or Am and treated with injection(s) of DTPA. Data on contamination events, subsequent treatments and bioassays were collected and stored in a database. Main results of the data analysis included:

- descriptive statistics on the 548 collected events of contamination and corresponding treatments protocols, which resulted in a total of 1158 injections,

- an evaluation of the increase of urinary excretion,

- a description of adverse reactions (cutaneous allergy) observed on only one case out of the 469 persons treated

- a common treatment protocol,

- a CEA report and several publications in peer-reviewed journals [1-5].

However, the systematic storage in this database of information on the monitoring and treatment of contaminated workers has been discontinued since the end of the first study in 2004. Still, while DTPA is the treatment of choice after Pu, Am and/or Cm contamination,

*Corresponding author: stephanie.lamart@cea.fr 
it is necessary to keep on updating statistics on contamination conditions, treatment protocols, bioassay results and eventual adverse reactions. This information is crucial to optimize treatment protocols and to possibly adapt the protocol on the contamination conditions. It is also a solid foundation for communicating with workers in case a treatment is recommended. Furthermore, the interpretation of excretion data after DTPA treatment remains a challenging step in the estimation of internal dose. In the meantime, biokinetic models from the International Commission for Radiological Protection does not account for DTPA treatment. Therefore, collection of excretion data affected by DTPA treatment can contribute to current efforts for developing a biokinetic model that includes the effect of DTPA.

As additional cases of contamination and DTPA treatments have been observed in France in the past 14 years, it was therefore decided to update this database and re-initiate a study including these new data. A working group composed of occupational physicians, biologists, pharmacists, experts in internal dosimetry and data analysists from the French nuclear industry, has been created early 2018. While the objectives will be similar as the initial study, the approach will be completed. Whereas the first study reported measurement results only based on urine data, the whole set of radiotoxicological and in vivo measurement will be used. In particular, faecal measurements will be included as DTPA also results in an increase of faecal excretion, whereas this effect has not yet been thoroughly evaluated. The increase of urinary and faecal excretion after DTPA will be quantified and evaluated depending on the radioisotope and route of entry. A numerical code based on the R-project for statistical computing (www.r-project.org) will be used to carry out this analysis which will facilitate the traceability of the results.

\section{References}

1. L. Grappin and P. Bérard, Radioprotection. 43(3): p. 465-466 (2008).

2. L. Grappin, P. Bérard, P. Beau, L. Carbone, X. Castagnet, C. Courtay, J. Le Goff, F. Ménétrier, M. Néron, and J. Piechowski. Rapport CEA-R-6097 (2006).

3. L. Grappin, P. Bérard, F. Ménétrier, L. Carbone, C. Courtay, X. Castagnet, J.P. Le Goff, M.O. Néron, P. Beau, and J. Piechowski, Radioprotection. 42(2): p. 163-196 (2007).

4. L. Grappin, P. Berard, F. Menetrier, L. Carbone, C. Courtay, X. Castagnet, J.P. Le Goff, M.O. Neron, and J. Piechowski, Radiat Prot Dosimetry. 127(1-4): p. 435-9 (2007).

5. L. Grappin, J.-P. Legoff, L. Carbone, C. Courtay, A.-L. Agrinier, M. Aninat, J.-C. Amabile, A. Florin, and F. Andre, Radioprotection. 44(4): p. 447-461 (2009). 